\title{
Macroeconometria e a estrutura a termo de taxas de juros - problemas (ainda) em aberto
}

\author{
Márcio Poletti Laurini*
}

\begin{abstract}
RESUMO - Nesta nota discutimos alguns problemas em aberto na estimação de modelos macroeconométricos que incorporam informações da estrutura a termo de taxas de juros. Em especial, discutimos algumas possíveis soluções para os problemas de identificação, máximos locais e dimensionalidade existentes nestes modelos e também formas de incorporar estruturas de parâmetros e volatilidades variantes no tempo em modelos econométricos de macrofinanças.
\end{abstract}

Palavras-chave: Estrutura a termo. Modelos de macrofinanças. Inferência.

\section{INTRODUÇÃO}

Uma tendência recente da literatura macroeconométrica é a construção de modelos macroeconômicos que incorporem informações da estrutura a termo de taxas de juros. O uso da estrutura a termo de taxas de juros em modelos macroeconométricos permite incorporar as expectativas futuras sobre a economia que estão contidas nos rendimentos observados para as diversas maturidades da curva de juros. O rendimento nominal exigido pelos investidores para títulos de distintas maturidades é um indicador importante sobre as expectativas de mercado de taxas de inflação, e indiretamente são indicadores antecedentes do ciclo econômico. Esta relação foi explorada na grande literatura que utiliza os spreads entre taxas de longo e curto prazo como indicadores antecedentes de inflação, crescimento do produto e consumo etc., como sumarizado em Estrella e Trubin (2006).

Os modelos mais recentes desta literatura, conhecidos como modelos de macrofinanças, não apenas tentam utilizar informações da estrutura a termo de taxas de juros como possíveis previsoras de variáveis macroeconômicas; nestes modelos as variáveis macroeconômicas e as variáveis financeiras, fundamentalmente medidas pela estrutura a termo de taxas de juros, são incorporadas em uma mesma estrutura de modelos fatoriais de precificação livre de arbitragem. Nestes modelos, preços de ativos e títulos são obtidos através de relações de não arbitragem entre as informações macroeconômicas e da curva de juros. O modelo básico desta literatura é o chamado modelo gaussiano-afim (affine gaussian), dado pela seguinte estrutura:

\footnotetext{
* Doutor em Estatística pela Universidade Estadual de Campinas. É professor adjunto do IBMEC-RJ. Endereço eletrônico: marcio.laurini@ibmecrj.br.
} 


$$
\begin{gathered}
\log M_{t}=-r\left(x_{t}\right)-\lambda\left(x_{t}\right)^{\prime} \varepsilon_{t+1}-1 / 2 \lambda_{t}^{\prime} \lambda_{t} \\
x_{t+1}=\Phi x_{t}+(I-\Phi) \mu+\sum \varepsilon_{t+1} \\
r_{t}=\rho_{0}+\rho x_{t} \\
\lambda_{t}=\lambda_{a}+\Lambda_{b} x_{t}
\end{gathered}
$$

onde $M_{t}$ é o chamado núcleo de precificação (pricing kernel), $x_{t}$ é um vetor de variáveis de estado não observadas, $r_{t}$ é a taxa nominal de juros de curto prazo, $\lambda_{t}$ é o preço de mercado do risco dos choques $\varepsilon_{t}, \Phi, \Sigma, \Lambda_{\mathrm{b}}$ são matrizes de parâmetros de dimensão $n$ por $n, \lambda_{\mathrm{a}}$ e $\rho_{0}$ são parâmetros escalares, $\rho$ e $\rho_{m}$ são vetores de parâmetros e o processo de choques $\varepsilon$ é assumido como normal multivariado ${ }^{1}$. Esta estrutura básica é tornada um modelo de macrofinanças pela adição de variáveis macro na especificação da taxa de juros de curto prazo. Por exemplo, no trabalho de Ang e Piazzesi (2003), o conjunto de variáveis macroeconômicas é derivado de uma regra de Taylor e inclui a inflação anualizada $\pi_{\mathrm{t}}$ e o gap do produto $G a p_{t}$, e assim a equação da curva de juros de curto prazo é dada por $r_{t}=\rho_{0}+\rho_{\pi} \pi_{t}+\rho_{g} G a p_{t}$.

Uma segunda classe de modelos utilizada na modelagem de macrofinanças são os modelos dinâmicos derivados da família de Nelson-Siegel. Estes modelos, introduzidos em Nelson e Siegel (1987) e generalizados em Diebold e Li (2006), podem ser utilizados como modelos de macrofinanças representando a estrutura a termo por uma equação de observação para os yields $y_{t}(\tau)$ observados em cada maturidade $\tau$ :

$$
y_{t}(\tau)=L_{t}+S_{t} \frac{1-e^{-\lambda \tau}}{\lambda \tau}+C_{t}\left(\frac{1-e^{-\lambda \tau}}{\lambda \tau}-e^{-\lambda \tau}\right)+\varepsilon_{t}(\tau)
$$

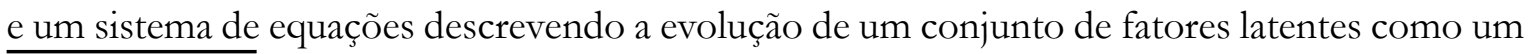
1 Nestes modelos, os preços de títulos são obtidos pela forma usual através do núcleo de precificação $P_{\tau, t}=E_{t}\left(P_{\tau-1}\right.$, $\left.{ }_{t+1} M_{t+1}\right)$, que possui uma forma afim dada por $P_{\tau, t}=E_{t}\left(A \tau+B^{\prime} x_{t}\right)$ e $A$ e $B$ são obtidos analiticamente como soluções de equações diferenciais ordinárias do sistema de equações:

$$
\begin{gathered}
0=\rho_{0}+A_{\tau}-A_{\tau-1}+1 / 2 B_{\tau-1}^{\prime} \sum \Sigma^{\prime} B_{\tau-1}-B_{\tau 1[(I-\Phi) \mu-\Sigma \lambda a]}^{\prime} \\
0_{n \times 1}=\rho+B_{\tau}-\left(\Phi-\Sigma \Lambda_{\mathrm{b}}\right)^{\prime} B_{\tau-1} \\
\text { S. } A . A_{0}=0, B_{0}=0_{\mathrm{n \times} 1}
\end{gathered}
$$


processo autorregressivo com a inclusão de variáveis macroeconômicas observadas; na aplicação de Diebold et al. (2006) este sistema é dado por:

$$
\left[\begin{array}{c}
L_{t} \\
S_{t} \\
C_{t} \\
C U_{t} \\
F F R \\
\pi_{t}
\end{array}\right]=\left[\begin{array}{c}
\mu_{L} \\
\mu_{S} \\
\mu_{C} \\
\mu_{C U} \\
\mu_{F F R} \\
\mu_{\pi}
\end{array}\right]+\Phi\left[\begin{array}{c}
L_{t-1} \\
S_{t-1} \\
C_{t-1} \\
C U_{t-1} \\
F F R_{t-1} \\
\pi_{t-1}
\end{array}\right]+\varepsilon_{t}
$$

com $C U_{t}$ denotando a capacidade utilizada da indústria, $F F R_{t}$ a meta de juros do Federal Reserve e $\pi_{t}$ a taxa de inflação. Esta estrutura derivada da formulação de Nelson-Siegel permite uma forma relativamente simples de unir uma representação de nível, inclinação e curvatura (Level, Slope and Curvature $)$ da curva de juros $\left(L_{t}, S_{t}\right.$ e $\left.C_{t}\right)$ com fatores macroeconômicos diretamente observados. Uma desvantagem desta classe de modelos é que ela não é uma representação livre de arbitragem, e, assim, não seria consistente com modelos de precificação em finanças. No entanto, um resultado importante, obtido por Christensen et al. (2009), Christensen et al. (2011) e depois generalizado em Joslin et al. (2011), é mostrar que a família de modelos de Nelson-Siegel pode ser transformada em um modelo afim de taxas de juros conforme a representação dada pelo sistema de Equações 1, com a inclusão de um termo de correção adicional, e desta forma é possível trabalhar com uma representação livre de arbitragem desta classe de modelos.

\section{PROBLEMAS NA ESTIMAÇÃO DE MODELOS DE MACROFINANÇAS}

Existem vários procedimentos de estimação disponíveis na literatura para a estimação dos modelos de macrofinanças apresentados acima. Podemos separar as metodologias básicas em três categorias - estimação usando condições de momentos, métodos baseados na função de verossimilhança e métodos baseados em simulações. Uma discussão aprofundada sobre o uso de métodos baseados em condições de momentos pode ser encontrada em Singleton (2006). Abordaremos rapidamente os métodos de verossimilhança e simulação.

Como a classe de modelos afins assume uma estrutura gaussiana, é possível formular diretamente a função de verossimilhança do processo para uma ampla classe de processos. Da mesma forma, a representação do modelo de macrofinanças baseada na representação de Nelson-Siegel de Diebold et al. (2006) é uma representação linear em espaço de estado, que permite a estimação por máxima verossimilhança através da decomposição do erro de previsão 
usando o filtro de Kalman. No entanto, a estimação por máxima verossimilhança é notoriamente problemática em modelos de estrutura a termo de taxas de juros, como relatado em Kim e Orphanides (2005). Eles apontam que esta classe de modelos é sujeita a problemas de múltiplos máximos locais e também problemas de identificação econométrica, o que significa que existe mais de um vetor de parâmetros que representa o mesmo máximo na função de verossimilhança, e estes distintos vetores de parâmetros podem ter interpretações econômicas completamente distintas. Nos artigos de Joslin et al. (2011) e Hamilton e Wu (2011) é possível solucionar os problemas de identificação e máximos locais na estimação de modelos afins de estrutura a termo de taxas de juros através de uma rotação na estrutura de fatores latentes. Esta rotação permite que parte dos parâmetros possa ser estimada eficientemente por mínimos quadrados ordinários. No entanto, a estimação por máxima verossimilhança também pode ser bastante complicada na presença de algumas estruturas de fatores latentes, como, por exemplo, a presença de volatilidade estocástica, e os métodos de Joslin et al. (2011) e Hamilton e Wu (2011) não podem ser ainda utilizados neste contexto.

Outro problema fundamental na estimação por máxima verossimilhança está na dimensionalidade do vetor de parâmetros. Por exemplo, na estimação do modelo de macrofinanças de Diebold et al. (2006), existem 42 parâmetros a serem estimados somente nas equações de estado, sendo necessário estimar ainda os parâmetros das matrizes de variância-covariância. Uma inspeção rápida na estimação deste modelo indica que ele não é identificado no máximo reportado em Diebold et al. (2006), e é possivel obter um máximo superior reiniciando o procedimento com distintos valores iniciais. Para tratar destes problemas de identificação e máximos locais, uma forma alternativa é o uso de métodos bayesianos. Em procedimentos de inferência bayesiana, o objetivo é recuperar a chamada distribuição posterior dos parâmetros e fatores latentes, que combina a informação da verossimilhança com a chamada distribuição a priori dos parâmetros, que condensa toda a informação subjetiva existente sobre o parâmetro antes de observar a amostra. Estes métodos de inferência bayesiana (e.g., Robert e Casella, 2005) podem ser implementados através dos métodos de simulação de Monte Carlo para cadeias de Markov, que consiste na simulação de processos que convergem para a distribuição posterior dos parâmetros e variáveis latentes.

Estes métodos bayesianos permitem tratar de problemas de dimensão elevada ao utilizar a informação a priori no processo de estimação, e também permitem resolver os problemas de identificação existentes em modelos de estrutura a termo de taxas de juros (e.g., Hamilton e Wu, 2011), já que o uso de informação a priori permite a estimação de modelos não identificados, como discutido em Caldeira et al. (2011). Estes métodos também evitam os problemas 
de múltiplos máximos locais, já que não é necessário utilizar procedimentos de otimização numérica, que são substituídos pelos procedimentos de simulação de cadeias de Markov. Eles também permitem incorporar estruturas de volatilidade estocástica, resolvendo um problema fundamental existente na geração atual de modelos de macrofinanças, dado pelo pressuposto simplificador que a volatilidade condicional das séries estudadas é constante no tempo, como apontado em Rudebusch (2008). A inclusão de estruturas de volatilidade estocástica pode alterar de forma significante as conclusões obtidas da estimação de modelos de macrofinanças.

Para tratar dos problemas de dimensionalidade e máximos locais, e adotar uma estrutura de volatilidade estocástica generalizada, em Laurini e Caldeira (2011) é utilizada uma metodologia de estimação bayesiana por simulações de Monte Carlo de cadeias de Markov de uma versão do modelo de macrofinanças de Diebold et al. (2006) com a inclusão de uma estrutura de volatilidade estocástica generalizada, utilizando uma estrutura similar à utilizada em Caldeira et al. (2011). Na estimação realizada em Laurini e Caldeira (2011) também é possível tratar o parâmetro de decaimento $\lambda$ como um fator latente variante no tempo, o que permite um melhor ajuste aos dados observados no tempo.

Dois resultados importantes podem ser observados. Esta metodologia de estimação permite um ganho significante de ajuste em relação ao modelo original de Diebold et al. (2006). Na Tabela 1 mostramos o erro médio e o desvio padrão dos erros obtidos pelo modelo estimado em Laurini e Caldeira (2011). As estatísticas colocadas em negrito mostram um ajuste melhor que o obtido pelo modelo de macrofinanças de Diebold et al. (2006, tabela 2 no artigo), mostrando que a nova estrutura de volatilidade estocástica consegue reduzir o erro médio e o desvio padrão do erro para boa parte das maturidades estudadas. Este resultado é explicado pela adição dos componentes de volatilidade estocástica, mas também pelo fato de que a metodologia de estimação bayesiana não sofre dos problemas de máximos locais que atingem as estimações por máxima verossimilhança, e assim consegue um resultado de ajuste superior.

TABELA 1 - AJUSTE - MODELO DE MACROFINANÇAS COM VOLATILIDADE ESTOCÁSTICA

\begin{tabular}{r|r|r}
\hline Maturidade & Erro médio & Desv. pad. \\
\hline 3 & $\mathbf{- 2 . 5 0 8}$ & $\mathbf{7 . 9 7 1}$ \\
6 & 1.202 & 6.542 \\
9 & $\mathbf{0 . 2 4 8}$ & 8.789 \\
12 & $\mathbf{0 . 1 6 6}$ & $\mathbf{8 . 9 4 1}$ \\
15 & $\mathbf{2 . 4 3 2}$ & $\mathbf{8 . 0 2 0}$ \\
18 & $\mathbf{2 . 4 6 9}$ & $\mathbf{7 . 1 3 4}$ \\
21 & 2.370 & $\mathbf{7 . 3 3 8}$ \\
24 & -1.991 & 7.277 \\
30 & -2.810 & 6.884 \\
36 & $\mathbf{- 3 . 1 5 7}$ & 6.806 \\
48 & $-\mathbf{1 . 6 8 8}$ & $\mathbf{9 . 6 5 4}$ \\
60 & -3.320 & 8.173 \\
\hline
\end{tabular}


TABELA 1 (CONTINUAÇÃO) - AJUSTE - MODELO DE MACROFINANÇAS COM VOLATILIDADE ESTOCÁSTICA

\begin{tabular}{r|r|r}
\hline Maturidade & Erro médio & Desv. pad. \\
\hline 72 & $\mathbf{1 . 6 8 9}$ & 9.584 \\
84 & $\mathbf{0 . 2 1 2}$ & $\mathbf{1 0 . 1 9 5}$ \\
96 & $\mathbf{2 . 9 0 2}$ & 9.694 \\
108 & $\mathbf{3 . 5 9 0}$ & $\mathbf{1 1 . 3 0 0}$ \\
120 & -1.858 & $\mathbf{1 2 . 4 0 0}$ \\
\hline
\end{tabular}

Um segundo resultado importante está na interpretação dos resultados obtidos. No modelo de macrofinanças de Diebold et al. (2006), o efeito dos choques é estudado através de uma função de resposta ao impulso derivada dos parâmetros do vetor autorregressivo (Equação 3). No Gráfico 1 mostramos a função de resposta ao impulso estimada, comparando o resultado original de Diebold et al. (2006) com o modelo com a inclusão dos componentes de volatilidade estocástica. Podemos notar que encontramos resultados significantemente diferentes para as respostas de curvatura à choques na inflação, inflação à capacidade utilizada e de inflação em relação à inflação passada, indicando que estruturas de volatilidade condicional são importantes e podem afetar a interpretação de modelos de macrofinanças.

\section{GRÁFICO 1 - FUNÇÃO DE RESPOSTA AO IMPULSO}

$\mathrm{L}$
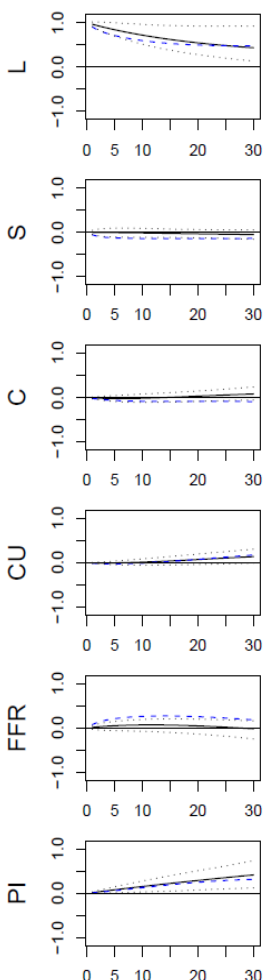

S
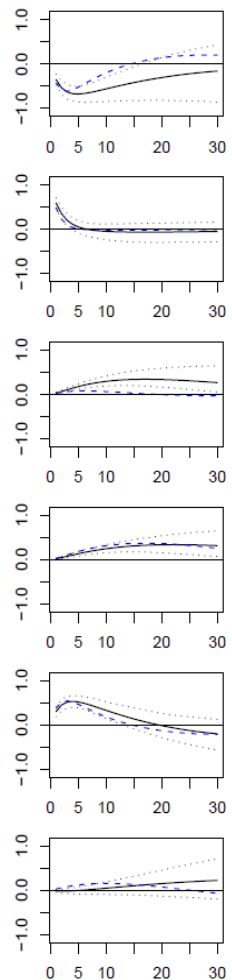

C
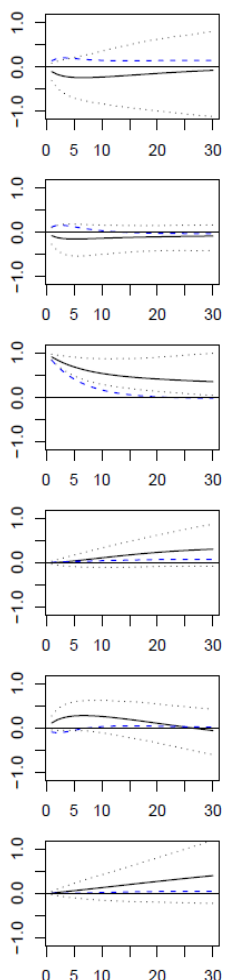

$\mathrm{CU}$
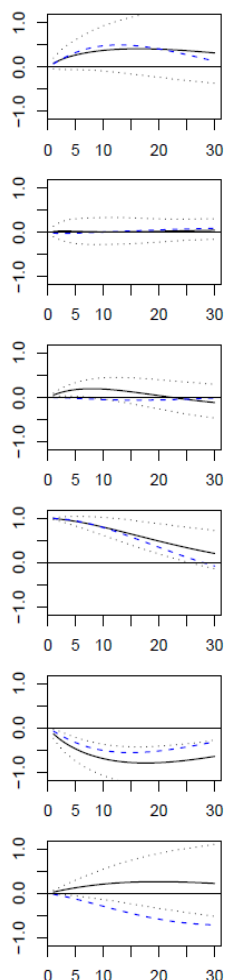

FFR
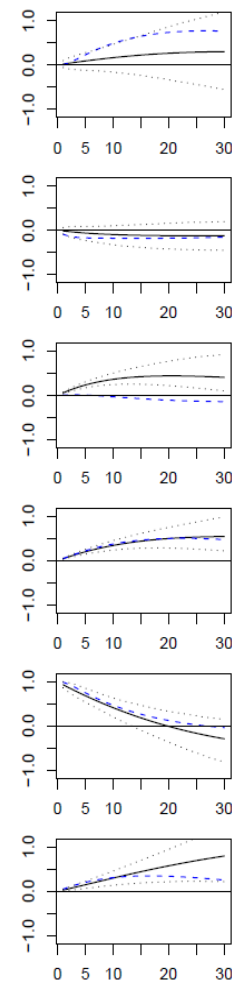

PI
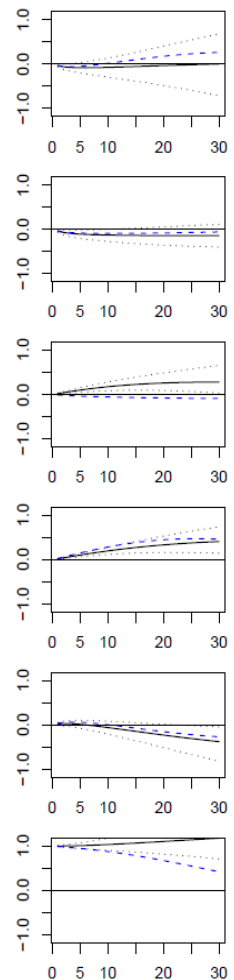

NOTA: Linha contínua: modelo com volatilidade estocástica; linha segmentada: modelo original; linhas pontilhadas: intervalo de credibilidade de $95 \%$. 


\section{CONCLUSÕES E EXTENSÕES}

A estimação de modelos de macrofinanças é um problema econométrico importante, já que os resultados obtidos por cada método de estimação podem ter interpretações econômicas distintas, como exemplificado na estimação anterior. Devido ao elevado número de parâmetros em modelos de macrofinanças, o uso de métodos bayesianos baseados em simulações de Monte Carlo de cadeias de Markov tem se consolidado como uma ferramenta fundamental para os procedimentos de inferência, em especial quando é necessária a estimação de fatores latentes, como os fatores de nível, inclinação e curvatura e fatores de volatilidade estocástica.

No entanto, o uso destes métodos de estimação ainda depende da aceitação da metodologia de inferência bayesiana, que pode ser criticada pelo uso de informação subjetiva (distribuições a priorı) na estimação. Contudo, é ainda possível utilizar estes métodos de estimação de simulações de Monte Carlo de cadeias de Markov como ferramentas para a estimação por máxima verosssimilhança. Um resultado importante é que a distribuição posterior dos dados em um procedimento de estimação bayesiana converge assintoticamente para o estimador de máxima verossimilhança, e é possivel utilizar métodos numéricos de aumentação do vetor de estados para recuperar este estimador (e.g., Jaquier et al., 2006), eliminando, assim, a influência da distribuição a priori na estimação de modelos de macrofinanças. Esta promissora abordagem ainda não foi explorada nesta literatura.

Outra abordagem que pode trazer resultados importantes nos procedimentos de estimação em modelos de macrofinanças é o uso de métodos bayesianos que utilizam aproximações analíticas em substituição aos métodos de simulação de Monte Carlo. Em Laurini e Hotta (2011), são utilizadas aproximações integradas de Laplace (Rue et al., 2009) para realizar inferência sobre parâmetros, fatores latentes e previsões para o modelo de Diebold e Li (2006). Esta metodologia permite estimações extremamente acuradas sem necessitar do uso de simulações, com um custo computacional reduzido. Esta classe de aproximações analíticas pode ser utilizada diretamente na classe de modelos gaussianos-afins, e assim também é outra importante metodologia ainda não explorada na estimação desta classe de modelos econométricos de finanças e macrofinanças.

\section{REFERÊNCIAS}

ANG, A.; PIAZZESI, M. A no-arbitrage vector autoregression of the term structure with macroeconomic and latent variables. Journal of Monetary Economics, v. 50, p. 745-787, 2003.

CALDEIRA, J. F.; LAURINI, M. P.; PORTUGAL, M. S. Inferência bayesiana aplicada ao modelo dinâmico de Nelson-Siegel com volatilidade estocástica nos fatores. Revista Brasileira de 
Econometria. 2011. No prelo.

CHRISTENSEN, J. H.; DIEBOLD, F. X.; RUDEBUSCH, G. D. An arbitrage-free generalized Nelson-Siegel term structure model. Econometrics Journal, v. 12, p. 33-64, 2009.

CHRISTENSEN, J. H.; DIEBOLD, F. X.; RUDEBUSCH, G. D. The affine arbitrage free class of Nelson-Siegel term structure models. Journal of Econometrics. 2011. No prelo.

DIEBOLD, F. X.; RUDEBUSCH, G.; ARUOBA, S. G. The macroeconomy and the yield curve: a dynamic latent factor approach. Journal of Econometrics, v. 131, p. 309-338, 2006.

DIEBOLD, F. X.; LI, C. Forecasting the term structure of government bond yields. Journal Of Econometrics, v. 130, n. 2, p. 337-364, 2006.

ESTRELLA, A.; TRUBIN, M. R. The yield curve as a leading indicator: some practical issues. Current Issues in Economics and Finance - Federal Researve Board of New York, v. 12, n. 5, p. 1-7, 2006.

HAMILTON, J.; WU, C. Identification and estimation in affine term structure models. 2011. Working paper não publicado.

JAQUIER, E.; JOHANNES, M.; POLSON, M. MCMC Maximum likelihood estimation for latent state models. Journal of Econometrics, v. 137, n. 2, p. 615-640, 2006.

JOSLIN, S.; SINGLETON, K. J.; ZHU, H. A new perspective on gaussian dynamic term structure models. Review of Financial Studies. 2011. No prelo.

KIM, D. H.; ORPHANIDES, A. Term structure estimation with survey data on interest rate forecasts. Board of Directors of Federal Reserve System - Finance and Economics Discussion Series, 2005.

LAURINI, M. P.; HOTTA, L. K. Forecasting the term structure of interest rates using integrated nested Laplace approximations. Ibmec RJ - Working paper, 2011.

LAURINI, M. P.; CALDEIRA, J. F. A stochastic volatility-macro-finance model. Trabalho em andamento.

NELSON, C. R.; SIEGEL, A. F. Parsimonous modelling of yield curves. Journal of Business, v. 60, n. 4, p. 473-489, 1987.

ROBERT, C. P.; CASELLA, G. Monte Carlo statistical methods. Springer, 2005.

RUDEBUSCH, G. Macro-finance models of interest rates and the macroeconomy. In: CONFERENCE OF FINANCIAL MARKETS AND REAL ACTIVITY, 2008, Banque de France.

RUE, H.; MARTINO, S.; CHOPIN, N. Approximated bayesian inference for latent gaussian models by using integrated nested Laplace approximations (with discussion). Journal of the Royal Statistical Society series B, v. 71, n. 2, p. 319-392, 2009.

SINGLETON, K. J. Empirical Dynamic Asset Pricing. New Jersey: Princeton University Press, 2006. 